\title{
Association of Maternal C-Reactive Protein in Severe Pre-Eclampsia
}

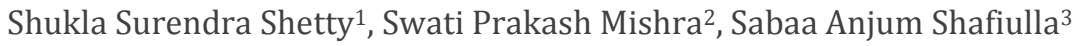 \\ ${ }^{1}$ Department of Obstetrics and Gynaecology, JJMMC, Davangere, Karnataka, India. \\ ${ }^{2}$ Department of Obstetrics and Gynaecology, JJMMC, Davangere, Karnataka, India. \\ ${ }^{3}$ Department of Obstetrics and Gynaecology, JJMMC, Davangere, Karnataka, India.
}

\section{ABSTRACT}

\section{BACKGROUND}

Endothelial cell dysfunction and inflammation are considered to have a role in the pathophysiology of pre-eclampsia(1).A generalized activation of circulating leukocytes (characteristic of inflammation) has been found during pre-eclampsia. Moreover, increased concentration of CRP and inflammatory markers has been reported in pre-eclampsia(2). We wanted to determine the serum CRP level in preeclampsia and assess the platelet dysfunction in pre-eclampsia and evaluate its association with severity of the disease.

\section{METHODS}

The study was conducted at a tertiary care centre from November 2015 to August 2017. The study included a total of 200 singleton pregnant patients. After collecting basic demographic data and brief history, detailed clinical examination was carried out, routine investigations along with serum $\mathrm{C}$ - reactive protein were done. Results were recorded and maternal and foetal outcomes were noted.

\section{RESULTS}

Among the 200 patients who were included in the study, it was found that majority of them were primigravidae. $88 \%$ of the patients showed increase in the C-reactive protein, which also correlated with the severity of the disease. Platelet count showed a decreasing trend with increasing severity of the disease and platelet distribution width showed an increasing trend with the increasing severity of the disease.

\section{CONCLUSIONS}

Serum C- reactive protein concentration increases in pre-eclampsia more than that compared to normal pregnancy. The serum C-reactive protein levels also helps in assessing the severity of the disease and its further prognosis. Further the abnormalities in platelet indices caused due to the disease process in pre-eclampsia is worsened due to thrombocytopenia. The morphological features of platelets as studied by Mean platelet volume and platelet distribution width are not consistent with the severity of the disease.

\section{KEY WORDS}

Severe Pre-Eclampsia, C Reactive Protein, Mean Platelet Volume, Platelet Distribution Width
Corresponding Author:

Dr. Shukla Surendra Shetty,

Professor and HOD,

Department of Obstetrics and Gynaecology, JJMMC, Davangere, Karnataka, India.

E-mail:Shukla.shetty@yahoo.com

DOI: $10.14260 / j e m d s / 2020 / 436$

How to Cite This Article:

Shetty SS, Mishra SP, Shafiulla SA. Association of maternal C-Reactive protein in severe pre-eclampsia. J. Evolution Med. Dent. Sci. 2020;9(28):2002-2006, DOI: $10.14260 / \mathrm{jemds} / 2020 / 436$

Submission 12-02-2020,

Peer Review 28-05-2020,

Acceptance 05-06-2020,

Published 13-07-2020.

Copyright (C) 2020 JEMDS. This is an open access article distributed under Creative Commons Attribution License [Attribution 4.0 International (CC BY 4.0)] 


\section{BACKGROUND}

Hypertensive disorders of pregnancy complicate about $8 \%$ of all gestations and are responsible for significant maternal and perinatal morbidity and mortality (3). Preeclampsia is a multisystem disorder of unknown origin characterized by blood pressure of more than $140 / 90 \mathrm{~mm}$ of $\mathrm{Hg}$ with or without proteinuria (4). It is the second leading cause of maternal mortality, (3) accounting for $16-18 \%$ of all maternal deaths. (5) Endothelial cell dysfunction and inflammation are considered to have a role in pathophysiology of pre-eclampsia (1). A generalized activation of circulating leukocytes (characteristic of inflammation) has been found during pre-eclampsia (6). Moreover, increased concentration of CRP and inflammatory markers has been reported in pre-eclampsia. C Reactive Protein is an acute phase protein, the levels of which rise in response to inflammation (7). C- Reactive Protein is synthesized by liver and it binds to phosphocholine expressed on the surface of dead and dying cells in order to activate the complement system (8). It is a member of pentraxin family of proteins.

Platelet abnormality is commonly identified haematological abnormality in preeclampsia (9). In addition to their important role in haemostasis and thrombosis, accumulating evidence demonstrates that platelets contribute to the inflammatory process, microbial host defence, wound healing angiogenesis and remodelling (10). Platelet indices such as Mean Platelet Volume (MVP) and Platelet Distribution Width (PDW) are found raised earlier than development of other recognized manifestations like hypertension or proteinuria when vascular changes occur $(11,12,13)$.

The present study was aimed to explore the effect of serum CRP concentration in pre-eclamptic women and its effect on pregnancy outcome and also to assess platelet dysfunction in severe PE and their association with severity of the disease.

\section{METHODS}

\section{Study Population}

This prospective study was carried out from November 2015 to August 2017 in the Obstetrics department of JJM medical college and hospitals attached to it. The source of this study were the pregnant women who were admitted with severe pre-eclampsia. This study was conducted on 200 patients. A written and informed consent was taken from those who satisfied the inclusion criteria.

Basis of Sample size:

Sample size $=\frac{z \alpha^{2} P(1-P)}{d^{2}}$

$\mathrm{z} \alpha^{2}$ - Std. normal variable

$\mathrm{d}$ - Absolute error

$P$ - Prevalence

e.g., Prevalence of pre-eclampsia in India - $5.4 \%$

Sample size- $3.8416 * 0.054 * 0.946 / 0.001=196.244 \cong 200$

\section{Inclusion Criteria}

1. Severe preeclampsia.
2. Singleton pregnancy.

3. Preterm, term and post term pregnancy.

\section{Exclusion Criteria}

1. Patients having underlying diseases.

- Diabetes mellitus.

- Renal disease.

- Chronic hypertension.

2. Symptomatic infectious diseases.

3. Patients having labour contractions and PROM and clinical chorioamnionitis.

Basic demographic data and brief history was collected at first. Clinical examination including evaluation of blood pressure and urine albumin was carried out. In all subjects, 5 $\mathrm{mL}$ of venous blood samples were drawn and assessed for the levels of CRP, Platelet count, Mean Platelet Volume and Platelet Distribution width.

\section{Determinations}

The sample for evaluating CRP was made to react with CRP latex reagent to form a precipitate which was measured turbidimetrically at $340 \mathrm{~nm}$. The MVP and PDW were measured by using a fully automated six part differential haematology analyser.

\section{Statistical Analysis}

Results were expressed in frequency and percentage for each category. Significance was assessed at $5 \%$ level of significance. Chi-square $/ 2 * 3$ Fischer extract test had been used to find the significance of study parameters on categorical scale.

1. p-Value of 0.05 or less was considered for statistical significance.

2. $\mathrm{p}$-Value of more than 0.05 was considered for statistical insignificance.

Results were recorded and the following maternal and foetal outcomes were noted-

1. Foetal outcome- birth weight.

2. Maternal outcome.

- Pulmonary oedema.

- Maternal mortality.

- ICU admission.

\section{Statistical Software}

The statistical software namely SPSS version 10 was used for the analysis of the data and Microsoft word and excel was used to generate graphs and tables.

\section{RESULTS}

The present study was conducted over a period of 24 months from September 2015 to August 2017 on 200 cases. These 200 patients fulfilled the criteria mentioned above. Venous blood samples were drawn from these patients and Serum CRP was estimated using an immune turbidimetric assay. Other 
variables assayed were MPV and PDW which were a part of the 3 part differential analyser.

In the study undertaken, the majority of the patients (88\%) showed an increase in CRP levels which correlates with the severity of the disease shown by $\mathrm{p}$ value of $<0.05$. The mean value of CRP being $13.9+/-4.3$. Platelet counts showed a decreasing trend in the present study which correlates with the increased severity of the disease. Mean platelet count being 1.65 lakhs/cubic mm. The MVP showed a normal trend in majority (93.5\%) of patients with increase in only $6.5 \%$ of patients. PDW showed an increasing trend in majority (73.5\%) of patients.

\begin{tabular}{|ccc|}
\hline Sl. No. & Variable & Value $\{$ Mean +/- SD $\}$ \\
1. & Age (in years) & $24.6+/-4.28$ \\
& Gravidity & 112 \\
2. & a. Primi & 88 \\
& b. Multi & $37.74+/-2.91$ \\
3. & Gestational age & \\
\hline \multicolumn{3}{r}{ Demographic Characteristics of Patients $(\mathbf{n}=\mathbf{2 0 0})$} \\
\hline
\end{tabular}

It was seen that the mean systolic blood pressure in 200 patients was $156.77 \mathrm{~mm}$ of $\mathrm{Hg}$ and the mean diastolic blood pressure was $105.43 \mathrm{~mm}$ of $\mathrm{Hg}$. In our study, the majority of patients with severe preeclampsia (88\%) had increased CRP levels and only $12 \%$ had normal CRP levels.

The relationship between severity of preeclampsia and platelet count, MPV was found to be insignificant. Out of 200 patients, $76.5 \%$ had platelet count of more than 2 lakhs/ cumm and only $1.5 \%$ had platelet count of less than 1 lakh/cumm. And also the majority (93.5\%) had normal MPV and only 13\% patients had increased MPV.

Among 200 patients, $73.5 \%$ had increased Platelet Distribution Width and only $26.5 \%$ had normal PDW.

\begin{tabular}{|ccc|}
\hline Sl. No. & Variable & Value $\{$ Mean +/- SD $\}$ \\
1. & Mean Blood Pressure & $156.77+/-\mathrm{mm} \mathrm{Hg}$ \\
& Systolic BP & $105.43+/-\mathrm{mm} \mathrm{Hg}$ \\
& Diastolic BP & $13.94+/-4.3 \mathrm{mg} / \mathrm{dl}$ \\
2. & C- Reactive Protein & $88 \%$ \\
& Increased & $12 \%$ \\
3. & Normal & $1.65 \mathrm{lakhs} / \mathrm{cumm}$ \\
& Platelet Count & $9.13+/-5.9 \mathrm{bcumm}$. \\
4. & Mean Platelet Volume & $93.5 \%$ \\
& Normal & $13 \%$ \\
& Increased & $16.38+/-1.79 \mathrm{fl}$ \\
5. & Platelet Distribution Width & $73.5 \%$ \\
& Increased & $26.5 \%$ \\
\hline \multicolumn{2}{c}{ Results of the Study } \\
\hline \multicolumn{2}{c}{ Normal } \\
\hline \multicolumn{2}{c}{} \\
\hline
\end{tabular}

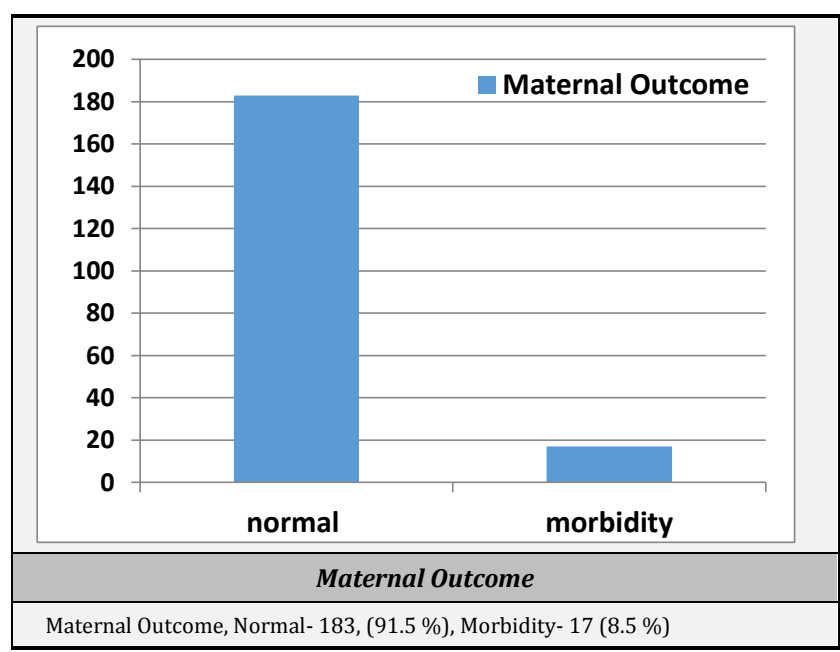

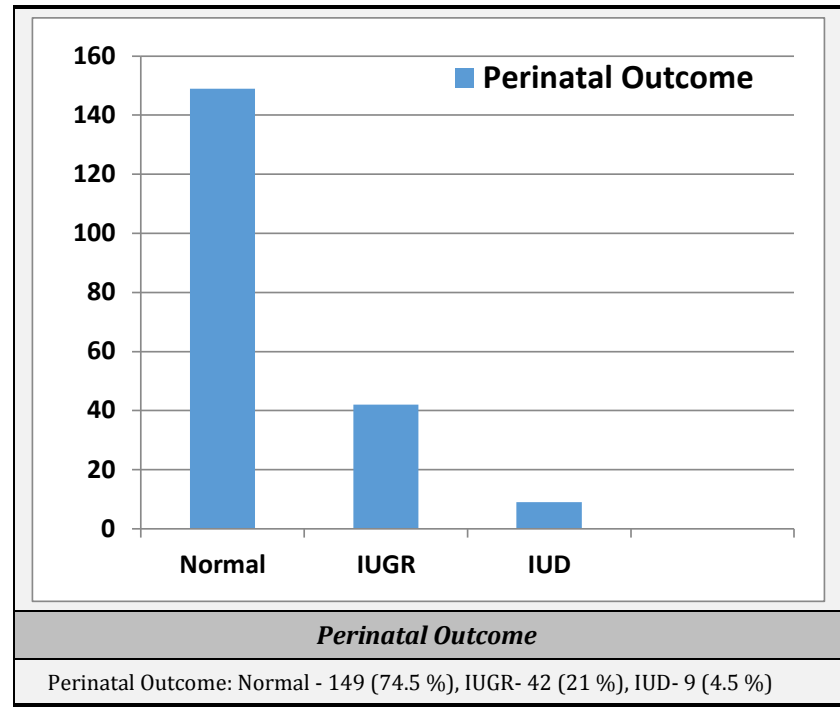

\section{Study Outcome}

Out of 200 patients, the majority (91.5\%) of the patients with severe preeclampsia had normal morbidity. Among 200 patients, the majority (74.5\%) had normal perinatal outcomes, and $21 \%$ had IUGR babies and $4.5 \%$ had Intrauterine foetal deaths.

\section{DISCUSSION}

Preeclampsia is a disease of pregnancy associated with endothelial cell damage. There is increasing evidence that preeclampsia is a systemic inflammatory disease.(2)

Studies have shown that markers of endothelial activation or inflammation have an active role in preeclampsia.(14) CRP, a sensitive marker of tissue damage and inflammation, was proposed to play a role in eliciting the inflammatory response characteristic of preeclampsia.(2)

C- reactive protein levels increase in normal pregnancy due to the presence of some amount of inflammation during pregnancy. CRP levels are higher around 28-37 wks. in cases. As the duration of pregnancy increases the CRP levels also increase and are higher in the range of preeclampsia than in normal pregnant women.

Soluble variants of the VEGF receptor 1 and of the Tumour Growth Factor $\beta$ coactivating receptor endoglin are involved in the antiangiogenic response, yet the inflammatory response is complex involving multiple cytokines. Clearly, cytokines such as Interleukin- 6 and TNF- $\alpha$ are elevated in pre-eclampsia (15). These cytokines support the expression of the acute phase protein C-Reactive Protein. CRP has also been found in amniotic fluid. This suggested local production sites, which were specified in the study by Parchim et al (8). As it binds to surface phosphocholine, it activates the complement system.

A positive relation has been observed between CRP levels and blood pressure (BP).Hence as CRP increases SBP and DBP also increases. There is a positive correlation between diastolic blood pressure and CRP level. The study conducted by E Teran, C Escudero et al (16) also showed similar CRP was significantly higher in preeclamptic women. Selhattin et al conducted a study to find the correlation of CRP with DBP. Urine albumin, other blood parameters of preeclampsia. This 
showed a strong positive correlation between serum CRP levels and DBP, urine protein excretion. Yusuf Ustan et al (2) study also showed significant correlation between MAP and CRP.

To identify the signalling pathway, the authors referred to the observation that CRP binds to phosphocholine. As the placenta is one of the tissues to which a phosphocholine transferase activity has been localized, posttranslational phosphocholine modification might apply (17).

As the neurokinin 3 receptor is thought to be also involved in hypertension in pregnancy and phosphocholine neurokinin B preferentially binds to this receptor, this pathway was explored. In the mice, neurokinin 3 receptor blockade improved the tissue lesions within the kidney and within the placenta, as well as soluble vascular endothelial growth factor receptor 1 levels on CRP exposure.

Knockdown of the phosphocholine transferase enzyme production resembled these findings. They found CRP and neurokinin B co-localized in villous syncytiotrophoblast cells and even identified a link between CRP, neurokinin 3 receptor signalling, and sFlt-1 secretion in human placental villous explants (17).

These findings provided a link between proinflammatory markers but also maternal conditions such as an elevated body mass index with enhanced CRP levels and development of preeclampsia. This is exciting because inflammation is considered a secondary event in most forms of preeclampsia, yet high body weight might arise as an independent initial event in the pathogenesis of preeclampsia as it predisposes to higher CRP levels, yet beyond pregnancy, if exposure to CRP is prolonged beyond acute infectious defense responses, and as more conditions and organs might be identified as phosphocholine transferase.

In the present study most of the $(64.5 \%)$ women belong to the 21-25 years age group, which is similar to the study conducted by Mirzaie et al. According to APMC 2008, frequency of disease increased with either end of maternal age, with a higher number of cases between 20-35 years.

In the present study $56 \%$ were primigravidae as compared to $44 \%$ multigravidae. According to Vitthal G Kuchaki, primigravidae show higher risk of preeclampsia.

In the present study women with preeclampsia present at lesser gestational age in labour or for induction of labour, with mean gestational age being $37.74+/-2.9$ weeks. These findings are in accordance with the study conducted by Kishwara et al.(18)

In this study the mean platelet count was 1.65 lakhs/cubic $\mathrm{mm}$. And the study describes that platelet consumption and aggregation increases as the disease gets more severe. In the study done by Tesfay et al. There was a significant gradual decrease in platelet count from normotensive pregnant women $(291.6+/-58.4 \mathrm{~mm})$ to mild preeclampsia group $(226+/-56.5 \mathrm{~mm})$ and severe preeclampsia group $(185.3+/-$ 60.2). Accordingly, a similar inverse relationship between platelet count and severity of preeclampsia was also documented by several researchers including Mohamed et al (19), Freitas et al (20), Kaito et al (21).

In the present study only $13 \%$ had increased MPV which was in accordance with study conducted by Cyehan et al (22) and Amita et al (23), which did not find a significant difference in the MPV between preeclampsia and normal pregnant group. Some researchers have suggested that platelet count and MPV cannot predict the risk of preeclampsia. However literature reveals conflicting results regarding the relation between MPV and preeclampsia.

In the present study, there was a significant increase in PDW in the severe preeclampsia group. These results were in agreement with that reported by Dadhich et al (24) who demonstrated a month wise increase in PDW in the PE group as compared to those in the normal pregnant group. These results are supported by Santos et al ${ }^{25)}$ and Nooh et al $(19,26)$. Rise in PDW serves as an important indicator of disease severity.

In the present study $88 \%$ of the patients with severe PE have increased CRP which correlates with the study done by Mirzaie et al, which clearly shows that serum CRP levels are higher in preeclampsia women. Determination of serum CRP levels in the third trimester pregnant women proved of great value in predicting the prognosis of preeclampsia.

\section{CONCLUSIONS}

Serum C- reactive protein concentration increases to a greater extent in pre-eclampsia when compared to that in normal pregnancy. Serum C- reactive protein levels also help in assessing the severity of the disease and its further prognosis Further the abnormalities in the platelet indices caused due to the disease process in pre-eclampsia is worsened due to thrombocytopenia. The morphological features of platelets as studied by Mean platelet volume and platelet distribution width are not consistent with the severity of the disease.

In the present study it was found that in majority of the patients, there is an increase in the C-reactive protein levels in patients with severe pre-eclampsia and there is a positive association of serum C-reactive protein with the severity of pre-eclampsia.

Although we could not find a significant association between the severity of pre-eclampsia and platelet dysfunction, the platelet count is found to decrease with increasing severity of the disease.

C- reactive protein has been proved as a good predictive marker for pre-eclampsia, and there is significant association with the severity of pre-eclampsia, maternal morbidity, and perinatal outcome.

\section{REFERENCES}

[1] Walsh SW. Pre-eclampsia: an imbalance in placental prostacyclin and thromboxane production. Am J Obstet Gynecol 1985;152(3):335-40.

[2] Ustun Y. Engin-Ustun Y, Kamaci M. Association of fibrinogen and $\mathrm{C}$ - reactive protein with severity of preeclampsia. Eur J Obstet Gynecol Reprod Biol 2005;121(2):154-8.

[3] Report of the National High Blood Pressure Education Program Working Group on High Blood Pressure in Pregnancy. Am J Obstet Gynecol 2000;183(1):S1-S22. 
[4] Fernando A. Hypertensive disorders in pregnancy. In: Practical guide to high risk pregnancy and delivery. $3^{\text {rd }}$ edn. Philadelphia: Mosby 1994: p.183-207.

[5] ACOG Committee on Obstetric Practice. ACOG practice bulletin. Diagnosis and management of preeclampsia and eclampsia. Number 33, January 2002. American College of Obstetricians and Gynaecologists. Int J Gynaecol Obstet 2002;77(1);67-75.

[6] Faas MM, Spaans F, De Vos P. Monocytes and macrophages in pregnancy and pre-eclampsia. Front Immunol 2014;5:298.

[7] Staff AC, Ranheim T, Khoury J, et al. Increased contents of phospholipids, cholesterol, lipid peroxidases in decidua basalis in women with pre-eclampsia. Am J Obstet Gynecol 1999;180(3 Pt 1):587-92.

[8] Parchim NF, Wang W, Iriyama T, et al. Neurokinin 3 receptor and phosphocholine transferase: missing factors for pathogenesis of C-reactive protein in preeclampsia. Hypertension 2015;65(2):430-9.

[9] Cunningham GF, Leveno KJ, Bloom SL, et al. Pregnancy hypertension. Williams Obstetrics. 24th edn. New York: McGraw-Hill Publication 2005: p. 706-14.

[10] Golebiewska EM, Poole AW. Platelet secretion: From haemostasis to wound healing and beyond. Blood Rev 2015;29(3):153-62.

[11] Vinodhini R, Lavanya K. Evaluation of platelet count as a prognostic index in eclampsia and pre-eclampsia. Int J of Modn Res Revs 2014;2(10):447-52.

[12] Hayashi M, Hamada Y, Ohkura T. Elevation of granulocyte colony stimulating factor in placenta and blood in preeclampsia. Am J Obstet Gynecol 2004;190(2):456-61.

[13] Fatemeh T, Marziyeh G, Nayereh G, et al. Maternal and perinatal outcome in nulliparous women complicated with pregnancy hypertension. J Pak Med Association 2010;60(9):707-10.

[14] Teran E, Escudero C, Moya W, et al. Elevated C- reactive protein and pro - inflammatory cytokines in Andean women with pre-eclampsia. Int J Obstet Gynecol 2001;75(3):243-9.

[15] Lamarca B. Endothelial dysfunction. An important mediator in the pathophysiology of hypertension during pre-eclampsia. Minerva Ginecol 2012;64(4):309-20.
[16] Hwang HS, Kwon JY, Kim MA, et al. Maternal serum highly sensitive C-reactive protein in normal pregnancy and preeclampsia. Int J Gynaecol Obstet 2007;98(2):105-9.

[17] C-reactive protein and its role in pre-eclampsia. https://www.ahajournals.org/toc/hyp/65/2

[18] Budak YU, Polat M, Huysal K. The use of platelet indices, plateletcrit, mean platelet volume and platelet distribution width in emergency non - traumatic abdominal surgery. A systematic review. Biochem Med (Zagreb) 2016;26(2):178-93.

[19] Nooh AM, Abdeldayem HM. Changes in platelet indices during pregnancy as potential markers for prediction of preeclampsia development. Open J of Obstet \& Gynecol 2015;5:703-12.

[20] Freitas LG, Alpoim PN, Komatsuzaki F, et al. Preeclampsia: are platelet count and indices useful for its prognosis? Hematology 2013;18(6):360-4.

[21] Kaito K, Otsubo H, Usui N, et al. Platelet size deviation width, platelet large cell ratio and mean platelet volume have sufficient sensitivity and specificity in the diagnosis of immune thrombocytopenia. $\mathrm{Br} \mathrm{J}$ of Hematol 2005;128(5):698-702.

[22] Ceyhan T, Beyan C, Baser I, et al. The effect of preeclampsia on complete blood count, platelet count and mean platelet volume. Ann Hematol 2006;85(5):320-2.

[23] Amita KR, Nithin KH, Shobha SN, et al. The role of platelet parameters as a biomarker in the diagnosis and prediction of severity of pre-eclampsia. Indian J Pathol Oncol 2015;2(2):57-60.

[24] Dadhich S, Agarwal S, Choudhary R, et al. Predictive values of platelet indices in development of preeclampsia. J South Asian Feder Obstet \& Gynecol 2012;4(1):17-21.

[25] Santos Dos EV, Jose MF. Measurement of platelet parameters in normal and pre eclamptic pregnant women. Rev Bras Gynecol Obstet 2004;26(3):201-6.

[26] Myatt L, Brewer AS, Langdon G, et al. Attenuation of the vasoconstrictor effects of thromboxane and endotheline by Nitric oxide in the human foetal-placental circulation. Am J Obstet Gynecol 1992;166(1 Pt 1):224-30. 